Annuaire suisse de politique de développement

26-2 | 2007

Financer le développement par la mobilisation des ressources locales

\title{
Quelques exemples d'engagements de la DDC dans le processus de décentralisation
}

\section{Pascal Raess}

\section{(2) OpenEdition}

\section{Journals}

Édition électronique

URL : http://journals.openedition.org/aspd/155

DOI : 10.4000/aspd. 155

ISSN : 1663-9669

Éditeur

Institut de hautes études internationales et du développement

Édition imprimée

Date de publication : 1 novembre 2007

Pagination : 247-248

ISBN : 978-2-88247-068-3

ISSN : 1660-5934

Référence électronique

Pascal Raess, «Quelques exemples d'engagements de la DDC dans le processus de décentralisation ", Annuaire suisse de politique de développement [En ligne], 26-2 | 2007, mis en ligne le 24 juin 2009, consulté le 08 septembre 2020. URL : http://journals.openedition.org/aspd/155 ; DOI https://doi.org/10.4000/aspd. 155 


\title{
Quelques exemples d'engagements de la DDC dans le processus de décentralisation
}

\author{
Pascal Raess
}

Bénin:

Appui au renforcement de l'Etat local dans le processus de décentralisation ${ }^{1}$

La politique de décentralisation au Bénin est inscrite dans la Constitution de 1990. Elle est mise en œuvre depuis les élections communales de décembre 2002 et janvier 2003 à travers un cadre législatif qui a pour objectifs essentiels la déconcentration des services de l'Etat aux niveaux départemental et communal, le transfert de compétences et de ressources aux organes communaux et infracommunaux et l'amélioration de l'offre de services publics de base par la prise en compte effective des besoins exprimés par les populations. Les difficultés majeures sont la maîtrise de leur rôle par les élus et leur capacité à coordonner les dynamiques locales existantes, en fournissant des résultats vérifiables, ainsi que le manque de disponibilité des ressources humaines et financières. Bien que propriétaires de la plupart des ouvrages publics, les communes s'investissent par exemple très peu dans leur gestion (entretien, réparation). La notion de "service public» est encore peu connue.

La DDC s'est impliquée dans la préparation de la décentralisation et dans le renforcement du développement local depuis 1997 à travers divers appuis. Son programme "Appui au renforcement de l'Etat local» suit une approche de développement des territoires, de promotion de la démocratie à la base et d'appui au niveau infracommunal. II veut améliorer les conditions de vie des femmes, des hommes et des enfants, instaurer des mécanismes favorisant la participation des acteurs de la société civile à la définition des politiques publiques locales et consolider le rôle de la commune comme moteur du développement local. Ce programme permet de mettre sur pied des synergies avec les autres programmes de la DDC (par exemple éducation ou économie locale). L'objectif principal peut être résumé de la manière suivante: la société civile, les opérateurs économiques et les communes se concertent au sein de "territoires de développement" pour fournir des services de bonne qualité et en quantité suffisante, répondant aux besoins des populations. Le programme est ambitieux; il sera mis en œuvre à travers les structures étatiques et le financement se fera selon les mécanismes de gestion des finances publiques. Par conséquent, les questions de financement de ces services en quantité et en qualité adéquates sont au cœur de ce programme.

\section{Mozambique: Programme de soutien à la décentralisation et à la municipalisation}

La décentralisation, la participation ainsi qu'un gouvernement local efficace constituent des défis majeurs dans l'effort du Mozambique de réduire substantiellement la pauvreté et donc de contribuer à atteindre les OMD. Le Plan d'action à long terme pour une réduction accélérée de la pauvreté (PARPA) du gouvernement du Mozambique ainsi que son plan complémentaire de réforme du secteur public affirment clairement que la décentralisation et la déconcentration favorisant des gouvernements locaux plus efficaces et transparents sont une condition nécessaire pour offrir des biens et des services publics de proximité aux populations démunies ainsi que pour générer de l'emploi et des revenus au niveau local. Ce défi demeure considérable dans un contexte de passé colonial, postcolonial et

1 Les informations relatives au Bénin, au Mozambique et au Pakistan sont tirées de documents internes de la DDC; celles relatives à la Bolivie sont basées sur la publication suivante: L. Thévoz, "La décentralisation dans les Andes ou l'art d'accompagner un processus ", Politorbis. Revue de politique étrangère, n 41, 2/2006, Berne, DFAE, 2006, pp. 43-50. 
postconflit caractérisé par une administration publique hautement centralisée et une négligence du niveau local en termes de ressources tant financières que humaines. Cependant, le gouvernement et le Parlement ont pris des décisions importantes depuis le début des années 1990 en vue de la décentralisation.

L'importance de ces ambitions décentralisatrices a été reconnue par la DDC quand, en 2002, elle a lancé un programme novateur de gouvernance locale, le «Programme de soutien à la décentralisation et la municipalisation". II est focalisé en particulier sur les aspects suivants: améliorer les procédures de planification (notamment budgétaires) en les rendant plus participatives, accroître les capacités pour une transparence et une redevabilité plus grandes de I'administration fiscale locale, améliorer I'offre et l'accessibilité de services publics notamment pour les populations marginalisées et contribuer au dialogue politique national sur la politique de décentralisation fiscale et sa mise en œuvre.

\section{Bolivie:}

\section{appui au processus de décentralisation}

Le processus bolivien de décentralisation peut faire état de toute une série d'innovations majeures qui ont fait date dans le domaine, dont notamment la méthode de "choc " (le pays a été municipalisé d'un jour à l'autre, le 1er juillet 1994, sans aucun projet pilote), I'institutionnalisation du contrôle populaire sur la gestion publique locale (par la reconnaissance des organisations sociales traditionnelles et l'attribution à celles-ci d'un réel droit de regard, elles sont devenues des partenaires incontournables des autorités municipales élues), le transfert direct de ressources financières nationales aux municipalités ainsi que la généralisation progressive du budget participatif.

La DDC appuie, de manière générale, les réformes de I'Etat qui peuvent amener une contribution particulièrement sensible à la réduction de la pauvreté. La décentralisation est l'une d'entre elles puisqu'elle favorise directement l'empowerment des acteurs locaux et, dans toute la mesure du possible, des plus défavorisés, la participation des acteurs locaux aux processus de décision et l'appropriation des réformes par ses propres bénéficiaires. Ensemble, ces conditions promeuvent l'accès des plus défavorisés à des services publics qui répondent à leurs besoins et à leurs moyens.

\section{Pakistan: programme innovateur de RFE}

Ce projet de la DDC entend tester, dans un contexte décentralisé au Pakistan, I'usage de l'instrument fiscal pour lutter contre la pauvreté et la destruction environnementale. La nouveauté de l'instrument implique une sensibilisation importante au sein des populations afin de les réunir autour d'un double principe: celui du "pollueur payeur» et de l'équité. En effet, les pauvres sont les populations les plus vulnérables aux catastrophes environnementales et à la destruction des écosystèmes. Ces destructions sont produites par des déficits de politiques économiques et sociales qui peuvent être corrigés.

II a été décidé d'intégrer cet instrument dans le processus de la gouvernance décentralisée. L'objectif est de renforcer le processus de décentralisation par l'utilisation d'instruments fiscaux appropriés pour l'environnement (changement des comportements) et pour la réduction de la pauvreté. Cela n'implique pas prioritairement I'introduction de nouveaux impôts ou taxes, mais la conduite de recherches sur la faisabilité de différents instruments de RFE dans le contexte pakistanais et sur leur mise en œuvre à travers des projets pilotes. De manière plus spécifique, les objectifs de ce projet novateur sont les suivants: faire accepter le principe du "pollueur payeur" au niveau du district, étudier la faisabilité de la mise en œuvre de différentes options dans des domaines tels que les coûts de la santé, de l'éducation, du logement ou de l'eau, améliorer les capacités pour l'utilisation d'instruments fiscaux ainsi que pour la formation de nouvelles coalitions avec la société civile sur ces questions. Les résultats ne seront donc pas en premier lieu l'augmentation des recettes fiscales, mais seront liés à la création d'un contexte favorable à la mise en place et à l'utilisation de l'outil fiscal pour le bien de l'environnement et la réduction de la pauvreté. 